\title{
The Effect of Twist Multiplier, Elastane Percentage and Pick Density on Denim Quality
}

\author{
A K Choudhary*, Sheena Bansal and Nikhil Lodha \\ Dr. B R Ambedkar National Institute of technology, India
}

*Corresponding author: A K Choudhary, Dr. B R Ambedkar National Institute of technology, Jalandhar - 144011, Punjab, India

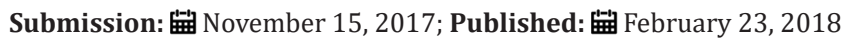

\begin{abstract}
For an end consumer of denim garments, the most important factors are performance and comfort after few aesthetic factors such as appearance and fashion appeal. For stretchable denim fabric, the fit related comfort lies in ability of the fabric to be stretched and recover when a repeated load is applied due to body movements without any permanent set called bagging. In this study, the interactive effect of twist multiplier of weft yarn, denier elastane fibre and fabric areal density on performance of denim fabric was investigated. The denier of elastane fibre used with weft yarn of three different twist multiplier- 4.9, 5.2 and 5.5, is changed as 40D, 55D and 70D. The areal density of fabric is changed through change in picks per inch as 50, 55 and 60. Performance was analysed on the basis of results of breaking strength, breaking elongation and cyclic loading stretch properties. The test results revealed that the tensile strength and breaking elongation properties of denim fabric, for both before and after cyclic loading are in the same trend, but after cyclic loading due to fatigue behaviour, tensile strength and breaking elongation was reduced. The breaking elongation increased as lycra content increases in the fabric and with increase in lycra content percentage, tensile strength was decreased slightly.
\end{abstract}

Keywords: Comfort; Performance; Denim; Elastane content; Areal density; Stretch; Recovery; Breaking strength; Twist multiplier

\section{Introduction}

Denim is sturdy cotton warp-faced textile with its demand in youngsters. The main factors affecting consumers when selecting garments are performance and comfort after few aesthetic factors such as appearance and fashion appeal. Performance as such is a very wide term and may range from satisfying the requirements during its use to the durability. Comfort is also considered by many consumers today as one of the performance requirements. Human skin can be stretched along with body movements [1]. Generally, the wearer also expects to see the same attitude from their clothing. Clothes are mostly under strain in some parts of the body, such as the knee, elbow and lower back areas [2,3]. Therefore, stretching of fabric is very important for the comfort of the wearer. Similarly for denim wear, the scope is increasing tremendously and recently the fashion trend is moving from denim to stretch denim (denim with Lycra) [4].

Stretch denim usually incorporates an elastic component (such as elastane) into the fabric to allow a degree of give in garments. Only a small percentage of spandex is required within the fabric (approx. 3\%) to allow a significant stretch capacity of around 15\%. Adding 1-5\% of Lycra with cotton will stretch the fabric over the body providing a more comfortable fit. The comfortable stretching of denim fabrics according to body movements as well as recovery after stretching, are most desirable properties. However, if clothes don't have such great flexibility; deformation occurs, generally dubbed as fabric bagging [2]. Rahman [5] studied the effect of spandex ratio on fabric physical and mechanical properties such as: breaking strength, breaking extension, shrinkage and fabric growth [5-9]. The findings of this study revealed that the ratio of Lycra had a significant influence on the physical properties of woven denim fabrics. Özdil [2] reported the stretch and bagging properties of denim fabrics containing different amounts of elastane. The test results revealed that increasing the amount of elastane in denim fabric enhanced comfort properties related to stretch.

The quest for highly stretchable fabrics with good and lasting handle properties such as absorbance, feel, stretch and comfort has inspired researchers to constantly involve in blending of natural and artificial fibres. One of the commonly used methods of blending is core yarn spinning. Superior mechanical properties of core spun yarn wider its application area. Core spun yarn primarily finds its application in industrial textile sector but during the last more than two decades it is being used in apparel sector primarily in denim. Stretch denims are made by using elastane core spun as weft in the fabric. Advantages of elastane core spun yarn are good recovery, better shape fitting and it facilitates ease of movement.

Denim fabrics made of core- spun yarns containing elastane gained great attention in the last decade due to its extensibility and comfort properties. Use of core-spun yarn containing lycra has become one of the effective ways to impart suitable stretch and 
recovery properties to fabrics without undesirable deformation of the garment during its service life. Fatigue due to cyclic loading is one of the most important factors in the non-conventional usages of textiles. Fatigue is the failure or decay of mechanical properties after repeated tensions. Fatigue tests give some information about the ability of material to withstand cracking which results in fracture after some cycles [10]. Shanbeh [11] had studied fatigue behaviour of core-spun yarns under cyclic tensile loading. They had observed that core spun yarn produced using the stronger multifilament yields higher yarn strength. Mourad et al. [12] had studied the Physical and Stretch Properties of Woven Cotton Fabrics Containing Different Rates of Spandex.

As the spandex content increases the contraction of the woven fabrics reacts in the same manner. As the amount of spandex increases, the tensile strength of the woven fabrics decreases. This is due to the lower tenacity of spandex fibers compared to cotton fibers. The fabric breaking elongation increases with the increase in spandex content. Das [13] had studied Fabric low-stress mechanical characteristics of elastane-cotton core-spun stretch yarns and fabrics. The increase in proportion of elastane core decreases the shear rigidity of fabric. The increase in elastane stretch reduces the bending hysteresis of fabrics. Qadir [14] studied the Effect of Elastane Denier and Draft Ratio of Core-Spun Cotton Weft Yarns on the Mechanical Properties of Woven Fabrics.

They had found out that by using a higher denier elastane in the core-spun cotton yarn, the fabric tear strength, stretch-ability and recovery after stretch increases but the fabric tensile strength decreases. Furthermore, by increasing the elastane draft ratio, the fabric tensile strength and stretch-ability increases whereas the fabric tear strength and recovery after stretch decreases. Many researchers have analysed the effect of change in elastane content on mechanical and comfort properties of different fabrics, but limited research has been carried out for change in areal density of denim fabric.

The present study is concerned with the tensile and elongation fatigue behaviour of stretchable denim which influenced by fabric parameters such as elastane content percentage and picks per inch. The study has been carried out using three different denier of elastane fibre used in core spun weft yarn and three level of fabric areal density with change in picks per inch (PPI). The effect of fabric parameters on fatigue behaviour is analysis by full factorial design and statistical techniques (ANOVA). It is revealed that, increase in cyclic elongation percentage the tensile strength and breaking elongation decrease due to bagging deformation. As elastane contents in fabric increases the tensile strength get reduced but breaking elongation increases. Bagging fatigue increases with increase in yarn twist multiplier.

\section{Material and Methods}

Denim fabric is made by different combination of ring and openend yarn (OE) such as ring/ring, OE/OE, and ring/OE. Weaving with combination of ring-spun yarn and open-end yarns can help to reduce fabric costs while still maintaining some favourable ring- spun fabric characteristics. So with this fact into consideration, our samples are also a combination of OE warp and core spun ring weft yarns with below specifications:

\section{Yarn specification}

Weft yarns of 16's Ne were produced from ring spinning system using three different elastane fibres of 40D, 55D and 70D at three TM level - 4.9, 5.2 and 5.5. Figure 1 shows a schematic of core spun yarn manufacturing at ring frame using elastane as the core fibre.

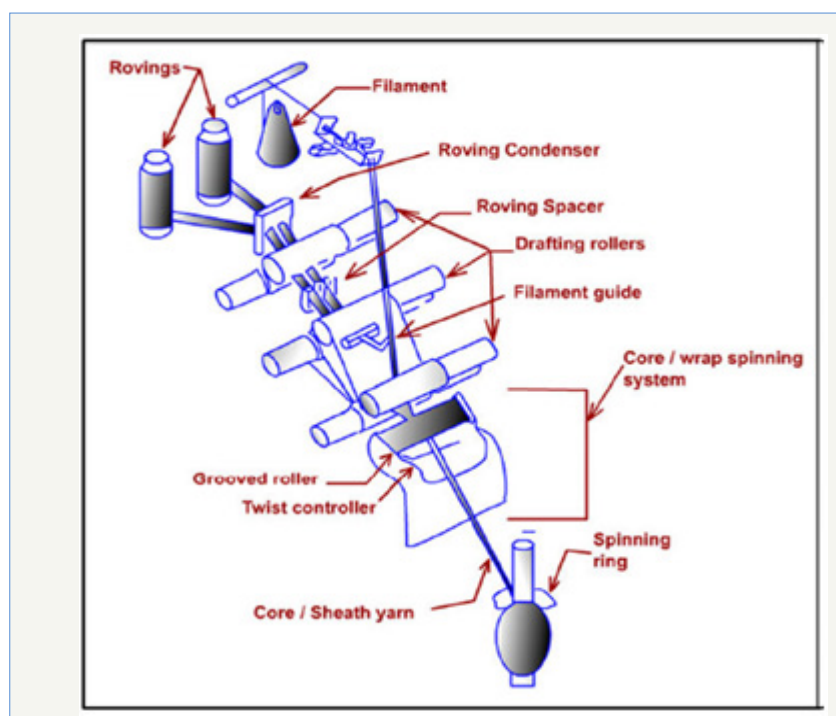

Figure 1: Manufacturing of core spun yarn in modified ring frame.

\begin{tabular}{|c|c|c|c|c|c|c|c|c|}
\hline \multicolumn{9}{|c|}{16 's Ne } \\
\hline & 4.9 & & & 5.2 & & & 5.5 & \\
\hline & & & & & & & & \\
\hline $40 \mathrm{D}$ & 55D & $70 \mathrm{D}$ & 40D & $55 \mathrm{D}$ & 70D & 40D & $55 \mathrm{D}$ & $70 \mathrm{D}$ \\
\hline 1 & 1 & 1 & 1 & 1 & 1 & 1 & 1 & 1 \\
\hline-50 & -50 & -50 & -50 & -50 & -50 & -50 & -50 & -50 \\
\hline-55 & -55 & -55 & -55 & -55 & -55 & -55 & -55 & -55 \\
\hline-60 & -60 & -60 & -60 & -60 & -60 & -60 & -60 & -60 \\
\hline
\end{tabular}

Figure 2: Flow chart of fabric specification.

\section{Fabric specification}

Denim fabric woven with 3/1 RHT twill weave was produced on air jet weaving machine which has weft insertion rate of 800 and reed spacing of 72.5 inch. The areal density of fabric was changed keeping ends density to be constant at 96 EPI and changing pick density from 50PPI, 55PPI and 60PPI. The warp count was 2/40's Ne with dark indigo colour. Figure 2 shows the total 27 denim fabric samples with three variations of each TM, Denier of elastane fibre and PPI. The denim fabric had been processed with light enzyme and softener to achieve finished ready to cut fabric for garment construction. 


\section{Testing condition}

The sample were tested under standard tropical atmospheric condition of $65 \pm 2 \% \mathrm{RH}$ and $27 \pm 2{ }^{\circ} \mathrm{C}$ temperature and the samples were conditioned for 24 hours before test.

\section{Tensile testing}

Zwick Universal tensile tester (BZ1-MM1450.ZW01) was used to measure the tensile properties. The ASTM D5035-06 standard test method had been used for breaking strength and elongation of textile fabrics (ravelled strip test) for which fabric samples of dimensions $300 \times 50 \mathrm{~mm}$ was prepared. The samples were subjected to elongation with gauge length $200 \mathrm{~mm}$ and cross head speed of $300 \mathrm{~mm} / \mathrm{min}$. Minimum 10 readings were taken for each sample to get the results significance at $95 \%$ confidence limit.

\section{Cyclic extension test}

Zwick universal tensile tester (BZ1-MM1450.ZW01) was used to measure the cyclic extension properties. The elongation was controlled by the sensor. Elongation cyclic test had been carried out at three different elongations $5 \%, 10 \%$ and $15 \%$ on all the 09 samples of denim fabrics. Samples were tested in weft direction under cyclic loading. In all set of experiments, the confidence interval selected $95 \%$. For cyclic extension fabric samples of dimensions $20 \mathrm{~cm} \times 5 \mathrm{~cm} \mathrm{~mm}$ was prepared. Zwick universal tensile tester was used with the following machine setting adjustment: gauge length $200 \mathrm{~mm}$, Cross head speed $300 \mathrm{~mm} / \mathrm{min}$. Extension was measured at 5\%,10\%, $15 \%$ after 20 cycles respectively for all samples. Approximately 5 readings were taken for each sample. After performing the cyclic loading, samples were relaxed for at least 30 min than their tensile strength and breaking elongation test had been carried out.

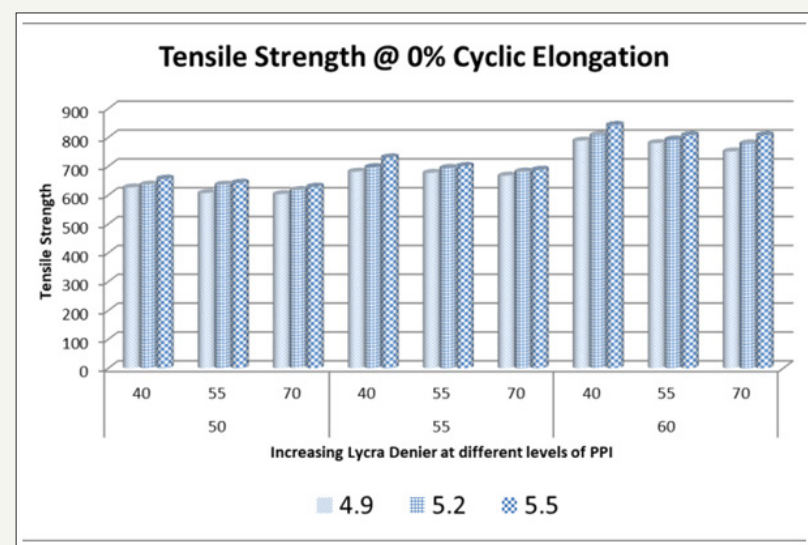

Figure 3: Influence of twist multiplier, lycra content $\%$ and pick density on tensile strength.

(a) Before cyclic extension.

\section{Results}

After conditioning of samples, the tests were performed under standard conditions to obtain the results for fatigue behaviour of denim fabrics i.e. Tensile strength and breaking elongation at $0 \%$, $5 \%, 10 \%$ and $15 \%$ cyclic elongation. The results were analysed through graphical and statistical methods. ANOVA was applied to know the interaction effect of three variables. The effect of factors in pair of two is shown through the graphical representation from Figure 3 (a to d) for Tensile strength and Figure 4 (a to d) for Breaking Elongation.

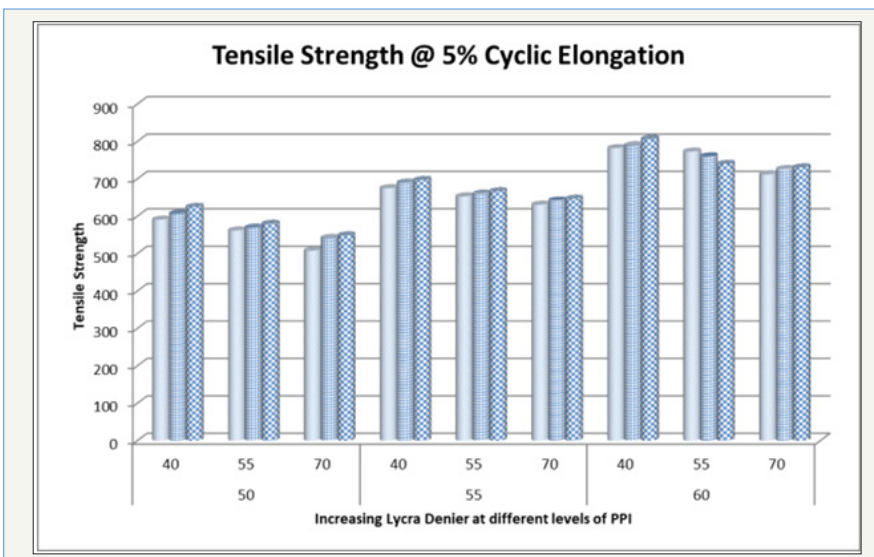

Figure 3: (b) After 5\% cyclic extension.

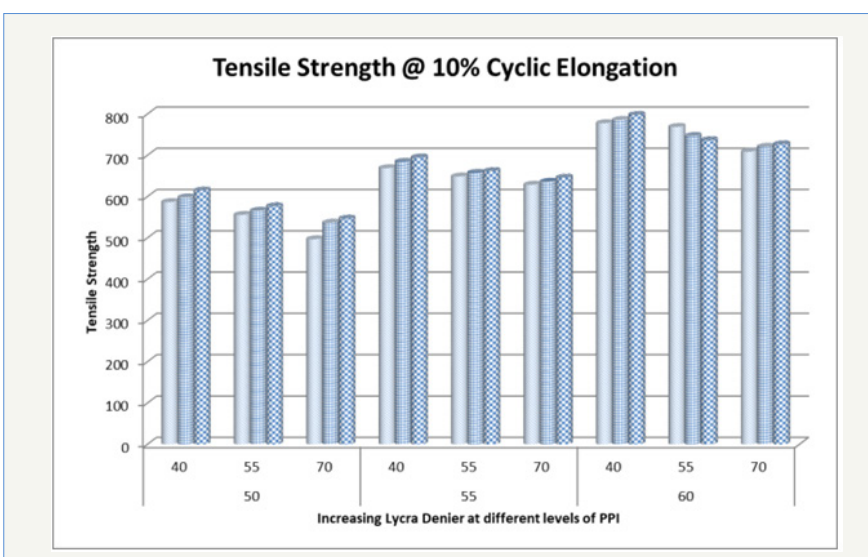

Figure 3: (c) After 10\% cyclic extension.

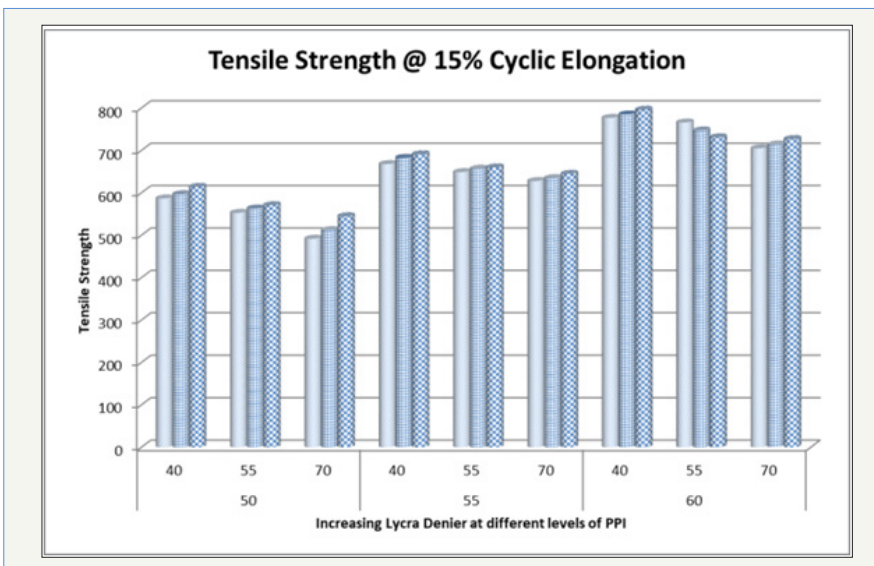

Figure 3: (d) After 15\% cyclic extension

\section{Discussions}

When a load was applied on a fabric, it gets distributed among the constituent yarns. First part of extension takes place due to crimp redistribution while the latter part is due to fibre extension. Such distribution of load is expected to be uniformly distributed all over the yarn structure in an ideal condition. But due to mass irregularity in staple fibre yarn however load sharing becomes 
non uniform. The high value for initial modulus of the fabric is probably due to frictional resistance to bending of the yarns. Once the frictional resistance is overcome, sharing of load continues till the structure failure. The failure generally takes place through the combination of breakage and slippage of fibre in the yarn which is manifested as a tensile strength of fabric. The breaking extension of a fabric is also a function of the extensibility of the component yarn and the waviness of yarn in fabric.

When fabric was subjected to cyclic extension the structure of the yarn got influenced. The change in structure of yarn is due to the slippage of sheath fibre. So the contribution of sheath fibre in load sharing got affected, which ultimately affected the tensile strength and breaking elongation of fabric.

\section{Influence of pick density (PPI) on tensile strength and breaking elongation}

Pick density increases the number of yarns per unit area providing more yarns for load sharing. So, the strength of fabric increases as PPI is increased. Similar trend has been observed from Figure 3 (a to d). It has been observed from the analysis of variance that the contribution of PPI on tensile strength is $87.4 \%$. For breaking extension, increase in PPI has adverse effect i.e. as PPI increases; more number of yarns will restrict the extension of fabric. Yarn to yarn friction is increased reducing mobility of yarns in fabric structure. This can also be said that the more number of yarns means less amount of resultant force per yarn. Thus breaking elongation is decreased as PPI is increased. It is observed from analysis of variance that the PPI has a significant contribution breaking extension with $17.55 \%$.

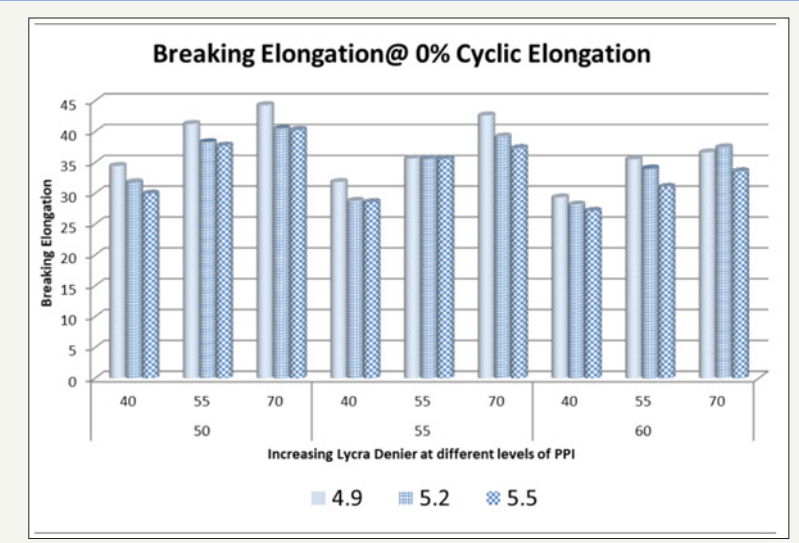

Figure 4: Influence of twist multiplier, lycra content $\%$ and pick density on breaking elongation.

(a) Before cyclic extension.

\section{Influence of Twist Multiplier (TM) on Tensile strength and breaking elongation}

Like ordinary ring yarn, twist has also significant effect on tenacity and extension of lycra core-spun yarn. With increase in yarn twist, tensile strength increases due an improvement of inter fibre cohesion in sheath and core of the yarn. When a lycra filament is introduced at the centre of the yarn, it supports additional load. At higher twist multiplier, yarn twist will be high and as a result, inter fiber cohesion at the sheath and that between core and sheath increases which facilitates better load bearing capability of the yarn leading to the increase in tensile strength. So this supports our observation from Figure 3 (a to d), the tensile strength of denim fabric increases as TM of weft core spun yarn has been increased. Through ANOVA, it is observed that TM has a significant effect on tensile strength. While in case of breaking elongation TM does not affect significantly as shown in Figure 4 (a to d).

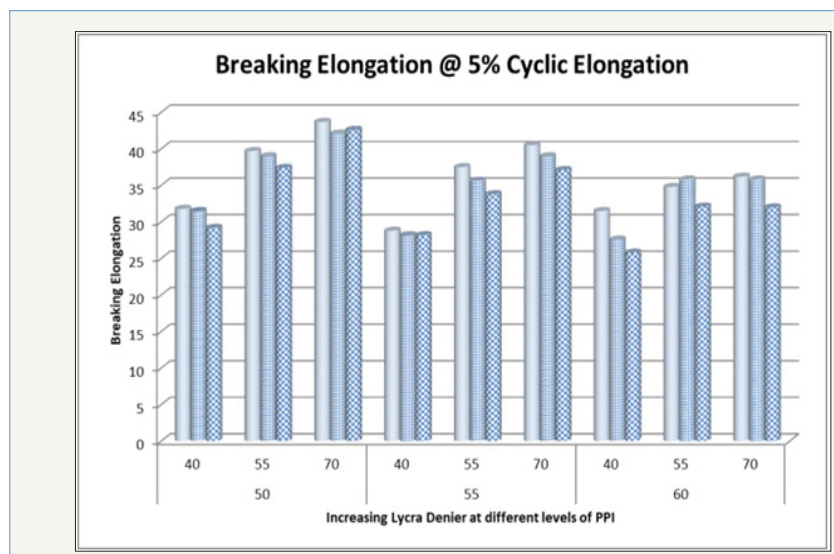

Figure 4: (b) After 5\% cyclic extension.

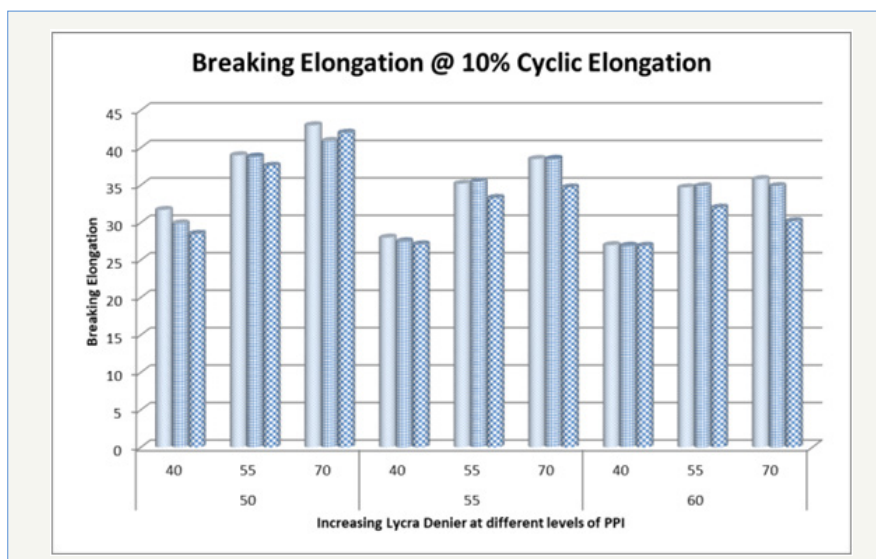

Figure 4: (c) After 10\% cyclic extension.

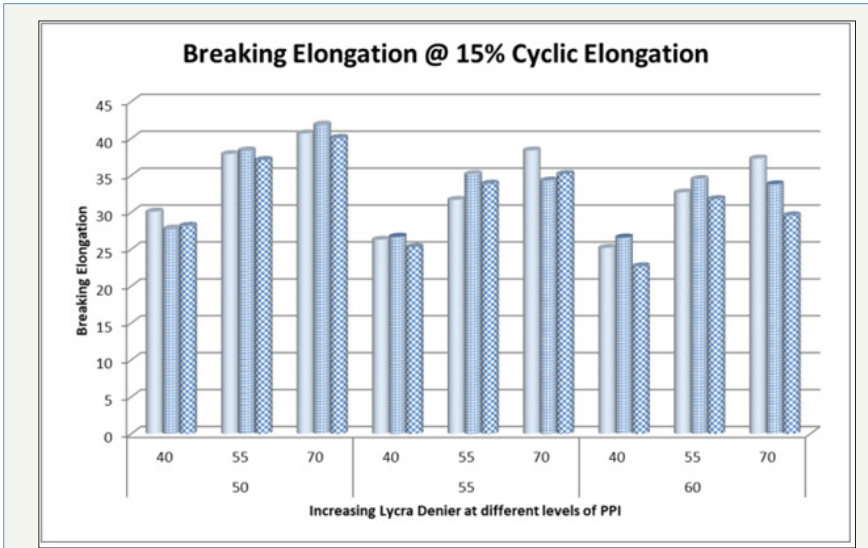

Figure 4: (d) After 15\% cyclic extension.

\section{Influence of denier of elastane fibre on Tensile strength and breaking elongation}

Since elastane has been used in core of the yarn, so the effect of elastane can be described in two ways - one is core to sheath 
interaction and another is low tenacity of elastane. Both of these effects are contradictory to each other, as elastane fibre in core helps sheath fibres to retain, but due to low tenacity of elastane fibre, the resultant tensile strength of fabric decreases. From Figure 3 (a to d), the acute decrease in tensile strength can be seen for increased denier of elastane fibre. For breaking elongation, elastane fibre helps the denim fabric for great stretch and recovery as shown in Figure 4 (a to d). Breaking elongation has $68.07 \%$ contribution as observed from ANOVA.

\section{Influence of cyclic extension on Tensile strength and breaking elongation}

It has been observed that tensile strength and breaking elongation properties of core spun denim fabric for both before and after cyclic extension shows same trend. With increase in the cyclic extension percentage the significance in the outcomes are higher due to increase in bagging fatigue behaviour. It is because fibre strands slippage is more than the fibre damage in yarn structure due to occurrence of plastic deformation in core spun yarn and thus in fabric. The change in structure of yarn is due to the slippage of sheath fibre so the contribution of sheath fibre in load sharing has reduced which ultimately reduces the tensile strength and breaking elongation.

\section{Conclusion}

Based on the investigation into the effect of twist multiplier, elastane percentage and pick density on denim quality, the following conclusions have been made:

i. The tensile strength and breaking elongation properties of denim fabric, for both before and after cyclic loading show the same trend. But after cyclic loading, due to fatigue behaviour tensile strength and breaking elongation is reduced. The maximum extension while functioning of denim garments should be less than maximum fabric extension to avoid growth and bagging behaviour of garments.

ii. The breaking elongation increases as lycra content increases in the fabric and with increase in lycra content percentage, tensile strength is decreased slightly. Garment extension and garment durability are inversely correlated as the higher lycra percentage decreases the strength of garments on repeated extension. Optimisation of required extension and garment longevity is desirable for new product development in range of body fit garments.

iii. Higher twist multiplier results in higher fabric tensile strength, but decrease in breaking elongation. The role of sheath fibres in yarn structure is very important for tensile strength of the denim fabric. So more the twist multiplier more is the hold of sheath fibres to the core elastane. Optimum level of extension can be achieved with higher twist multiplier with high strength of denim garments after a number of uses.

iv. Increased pick density, increases the tensile strength and breaking elongation is reduced. Higher pick density indicates more compact structure. So due to a compact structure less porous denim fabrics used in winter wear can provide an advantage of high strength and durability with optimum level of extension.

\section{References}

1. Kumar S, Chatterjee K, Padhye R, Nayak R (2016) Designing and development of denim fabrics: Part 1-Study the effect of fabric parameters on the fabric characteristics for women's wear. Journal of Textile Science \& Engineering 6: 265.

2. Özdil N (2008) Stretch and bagging properties of denim fabrics containing different rates of elastane. Fibres and Textiles in Eastern Europe 66(1): 63-67.

3. Mehta VP (1992) An introduction to quality control for the apparel industry. ASQC, Quality Press, New York, USA, pp. 79-83.

4. Kumar V, Nayak R (2014) Sewing performance of PV and PES air-jet textured sewing threads in denim fabrics. Journal of Textile and Apparel Technology and Management 8(4): 1-12.

5. Rahman 0 (2011) Understanding consumers' perceptions and buying behaviours: Implications for denim jeans design. Journal of Textile and Apparel Technology and Management 7(1): 1.

6. Lee JT, Kim MW, Song YS, Kang TJ, Youn JR (2010) Mechanical properties of denim fabric reinforced poly (lactic acid). Fibers and Polymers 11(1): 60-66.

7. Haghighat E, Etrati SM, Najar SS (2013) Modelling of needle penetration force in denim fabric. International Journal of Clothing Science and Technology 25(5): 361-379.

8. Hua T, Tao XM, Cheng KPS, XU BG, Huang XX (2013) An experimental study of improving fabric appearance of denim by using low torque singles ring spun yarns. Textile Research Journal 83(13).

9. Sariisik M (2004) Use of Cellulases and their effects on denim fabric properties. AATCC review 4(1): 24-29.

10. Șengöz NG (2004) Bagging in textiles. Taylor \& Francis Textile Progress 36(1): 1-64.

11. Shanbeh M, Hossein H, Fatemeh Y M (2012) An investigation into the fatigue behaviour of core-spun yarns under cyclic tensile loading. Journal of Engineered Fabrics \& Fibers (JEFF) 7(4): 95-103.

12. Mourad MM, Elshakankery MH, Alsaid AA (2012) Physical and stretch properties of woven cotton fabrics containing different rates of spandex. Journal of American Science 8(4): 567-572.

13. Das A, Chakraborty R (2013) Studies on elastane-cotton core-spun stretch yarns and fabrics: Part II-Fabric low-stress mechanical characteristics. Indian Journal of Fibre \& Textile Research 38(4): 340348 .

14. Qadir, Bilal, Tanveer H, Mumtaz M (2012) Effect of elastane denier and draft ratio of core-spun cotton weft yarns on the mechanical properties of woven fabrics. Journal of Engineered Fibers \& Fabrics (JEFF) 7(4): 23-31. 


\section{Your subsequent submission with Crimson Publishers} will attain the below benefits

- High-level peer review and editorial services

- Freely accessible online immediately upon publication

- Authors retain the copyright to their work

- Licensing it under a Creative Commons license

- Visibility through different online platforms

- Global attainment for your research

- Article availability in different formats (Pdf, E-pub, Full Text)

- Endless customer service

- Reasonable Membership services

- Reprints availability upon request

- One step article tracking system 